\title{
NUITAH ZOPHEI SWADESH LIST
}

\author{
October, 2020 \\ James C. Wamsley, Kimberly Sakhong, Samson Lotven ${ }^{1}$ \\ ${ }^{1}$ Indiana University Department of Linguistics
}

\section{INTRODUCTION}

This paper presents a preliminary 100-item Swadesh word list for Nuitah Zophei. Zophei or Zyphe (ISO 639-3 ZYP) belongs to the Maraic branch of Kuki-Chin within the Tibeto-Burman language family (Eberhard et. al, 2020). Nuitah (also known as Leitak) is a village in the southern part of the Zophei-speaking area in Thantlang Township, Chin State, Myanmar. The word list comes from the intuitions of our co-author Kimberly Biakthapar Sakhong, a 21-year-old native speaker born in Nuitah village of Zophei parents (both from Nuitah), who lived for some of her childhood in Hakha City (in Burma/Myanmar) and Malaysia before moving to the US. She currently lives in Indianapolis, Indiana. She also speaks Hakha Lai, Senthang, and English. The Nuitah Zophei consonant and vowel inventories are as follows. Note that we use a superscript $[\mathrm{h}]$ after sonorants to indicate voicelessness, e.g. $[\mathrm{m}]$ is a voiced nasal and $\left[\mathrm{m}^{\mathrm{h}}\right]$ is its voiceless counterpart.

$/ \mathrm{b} p \mathrm{p}^{\mathrm{h}} \mathrm{dt} \mathrm{t}^{\mathrm{h}} \mathrm{k} \mathrm{k}^{\mathrm{h}} \mathrm{v} \mathrm{s} \mathrm{zh} \widehat{\mathrm{ts}} \widehat{\mathrm{ts}}^{\mathrm{h}} \widehat{\mathrm{tl}} \widehat{\mathrm{tl}}^{\mathrm{h}} \mathrm{m} \mathrm{m}^{\mathrm{h}} \mathrm{n} \mathrm{n}^{\mathrm{h}} \mathrm{y} 1 \mathrm{l}^{\mathrm{h}} \mathrm{I} \mathrm{I}^{\mathrm{h}}$ ?/

/i ii e ee a aa o oo u uu it $u$ tu ai oi ui ia ua/

In addition, Nuitah Zophei has three register tones, high, mid, and low, represented orthographically with diacritic markings for high tone (á) and low tone (à) and no marking for mid tone. Section 2 includes a 102-item Nuitah Zophei word list based on Swadesh's (1971) 100-word list (multiple notable translations of some entries are included).

\section{WORD LIST}

\begin{tabular}{|c|l|l|}
\hline & \multicolumn{1}{|c|}{ Gloss } & \multicolumn{1}{c|}{ Nuitah Zophei } \\
\hline 1. & I & ka máá \\
\hline 2. & You & na máá \\
\hline 3. & We & kaa nút \\
\hline 4. & this & máá he? \\
\hline $5 a$. & that (medial) & máá khi? \\
\hline $5 b$. & that (distal) & máá k ${ }^{\text {ha? }}$ \\
\hline 6. & who (subj/obj) & a huu / a húú \\
\hline 7. & what (subj/obj) & zai / záí \\
\hline 8. & not that & min ii \\
\hline 9. & all (of a number) & a zoo púí tíí aa \\
\hline
\end{tabular}


Indiana Working Papers in South Asian Languages and Cultures

\begin{tabular}{|c|c|c|}
\hline 10. & many & lon tii $^{1}$ \\
\hline 11. & one & mee $\mathrm{k}^{\mathrm{h}} \mathrm{e} ?$ \\
\hline 12. & two & mee $\mathrm{n}_{\text {hij }}$ \\
\hline 13. & big & lén \\
\hline 14. & long (not wide) & soi \\
\hline 15. & small & $\mathrm{ts}^{\mathrm{h}} \mathrm{ia}$ \\
\hline 16. & woman & tsa nu? \\
\hline 17. & $\operatorname{man}$ & tsa poo \\
\hline 18. & person & tsòy soo \\
\hline 19. & fish & yoo \\
\hline 20. & bird & pa vóó \\
\hline 21. & $\operatorname{dog}$ & ui \\
\hline 22. & louse & $\mathrm{I}^{\mathrm{h}} \mathrm{i}$ ? \\
\hline 23. & tree & 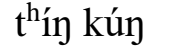 \\
\hline 24. & seed & tsii \\
\hline 25. & leaf & ts $^{h_{\text {in }} \mathrm{n}^{\mathrm{h}} \text { óó }}$ \\
\hline 26. & root & a I Ihág \\
\hline 27. & bark (of a tree) & hòy \\
\hline 28. & skin & vín \\
\hline 29. & flesh & sa ba tún \\
\hline 30. & blood & $\mathrm{t}^{\mathrm{h} y \mathrm{f}}$ \\
\hline 31. & bone & Itúú \\
\hline 32. & grease & a t thóí $^{2}$ \\
\hline 33. & egg & aa tui \\
\hline 34. & horn & kii \\
\hline 35. & tail & a la mª́ái \\
\hline 36. & feather & ma $\widehat{\mathrm{tl}^{\mathrm{h}}} \mathrm{Oo}$ \\
\hline 37. & hair & sán \\
\hline 38. & head & $\mathrm{lu}$ \\
\hline 39. & ear & noo \\
\hline 40. & eye & $\mathrm{mi}$ ? \\
\hline 41. & nose & $\mathrm{n}^{\text {hóó }}$ \\
\hline 42. & mouth & koo \\
\hline 43. & tooth & hòò \\
\hline 44. & tongue & lùì \\
\hline 45. & nail/claw & ba tín \\
\hline
\end{tabular}

\footnotetext{
${ }^{1}$ Unlike other Zophei varieties, Nuitah Zophei exhibits regressive nasal assimilation, so for example, <lon tii $>$ is pronounced [lontii]. This place assimilation yields allophony in surface realization, though there are no phonemic place contrasts in final nasals.
}

IWPSALC 2(1):40-43 
Indiana Working Papers in South Asian Languages and Cultures

\begin{tabular}{|c|c|c|}
\hline 46. & foot & $\mathrm{p}^{\text {háí }}$ \\
\hline 47. & knee & $\mathrm{k}^{\mathrm{h} u ̀ ?}$ \\
\hline 48. & hand & kì? \\
\hline 49. & belly & ven \\
\hline 50. & neck & .ón \\
\hline 51. & breasts & méé méé \\
\hline 52. & heart & $\mathrm{t}^{\mathrm{h}}$ ín \\
\hline 53. & liver & lùy \\
\hline 54. & drink & din \\
\hline 55. & eat & ai \\
\hline 56. & bite & toi \\
\hline 57. & see & $\mathrm{m}^{\mathrm{h}} \mathrm{ú}$ ? \\
\hline 58. & hear & 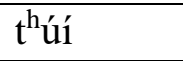 \\
\hline 59a. & know & bii \\
\hline $59 \mathrm{~b}$. & know & $\mathrm{n}^{\mathrm{h}} \mathrm{y}_{\mathrm{it}} / \mathrm{n}^{\mathrm{h}} \mathrm{i}$ ? \\
\hline 60. & sleep (same as 'lie') & í? / íí \\
\hline 61. & die & $\mathrm{t}^{\mathrm{h}} \mathrm{i} / \mathrm{t}^{\mathrm{h}} \mathrm{ii}$ \\
\hline 62. & kill & $\mathrm{t}^{\mathrm{h}} \mathrm{Oo} / \mathrm{t}^{\mathrm{h}} \mathrm{e}$ ? \\
\hline 63. & swim & tui loi \\
\hline 64. & fly & zoi \\
\hline 65. & walk & síí \\
\hline 66. & come & va sii \\
\hline 67. & lie & í? / íí \\
\hline 68. & sit & toi \\
\hline 69. & stand & doo \\
\hline 70. & give & pe?/pii \\
\hline 71. & say & dii \\
\hline 72. & sun & nii \\
\hline 73. & moon & $\widehat{t}^{\mathrm{h}} \mathrm{OO}$ póó \\
\hline 74. & star & aa súf \\
\hline 75. & water & tui \\
\hline 76. & rain & $\mathrm{k}^{\mathrm{h}} \mathrm{ua}$ suu \\
\hline 77. & stone & a lun \\
\hline 78. & sand & pa sè? \\
\hline 79. & earth & á lúí \\
\hline 80. & cloud & á máí \\
\hline 81. & smoke & $\mathrm{a} \mathrm{k}^{\mathrm{h}} \mathrm{ùù}$ \\
\hline 82. & fire & máí \\
\hline 83. & ashes & máí caay \\
\hline
\end{tabular}


Indiana Working Papers in South Asian Languages and Cultures

\begin{tabular}{|c|c|c|}
\hline 84. & to be burnt/hot & kááy \\
\hline 85. & path & laay \\
\hline 86. & mountain & tlày \\
\hline 87. & red & a sèn \\
\hline 88. & green & a $x^{h} i ̀ n$ \\
\hline 89. & yellow & ai $\mathrm{d}^{\mathrm{h}} \mathrm{h}_{1 i ́}$ \\
\hline 90. & white & a ıày \\
\hline 91. & black & a vòn \\
\hline 92. & night & zéén \\
\hline 93. & hot & se? \\
\hline 94. & cold & kóó \\
\hline 95. & full & $\widehat{t s}^{\text {hi? }}$ \\
\hline 96. & new & $\mathrm{t}^{\mathrm{h}} \mathrm{aa}$ \\
\hline 97. & good & tsha? \\
\hline 98. & round & pún \\
\hline 99. & dry & Ióí \\
\hline 100. & name & $\min$ \\
\hline
\end{tabular}

\section{REFERENCES}

Eberhard, D. M., Simons, G. F., and Fennig, C. D. (eds.) (2020) "Ethnologue: Languages of the World. Twenty-second edition” (SIL International, Dallas, Texas), Online version: http://www.ethnologue.com.

Swadesh, M. (1971) The origin and diversification of language (Aldine-Atherton, Chicago, Illinois), pp. 1-350. 